\title{
TOPOLOGICAL MODEL CATEGORIES GENERATED BY FINITE COMPLEXES
}

\author{
ALEX CHIGOGIDZE AND A. KARASEV
}

\begin{abstract}
Our main result states that for each finite complex $L$ the category TOP of topological spaces possesses a model category structure (in the sense of Quillen) whose weak equivalences are precisely maps which induce isomorphisms of all $[L]$-homotopy groups. The concept of $[L]$-homotopy has earlier been introduced by the first author and is based on Dranishnikov's notion of extension dimension. As a corollary we obtain an algebraic characterization of $[L]$-homotopy equivalences between $[L]$-complexes. This result extends two classical theorems of J. H. C. Whitehead. One of them - describing homotopy equivalences between $\mathrm{CW}$-complexes as maps inducing isomorphisms of all homotopy groups - is obtained by letting $L=\{$ point $\}$. The other - describing $n$-homotopy equivalences between at most $(n+1)$ dimensional $\mathrm{CW}$-complexes as maps inducing isomorphisms of $k$-dimensional homotopy groups with $k \leq n$ - by letting $L=S^{n+1}, n \geq 0$.
\end{abstract}

\section{INTRODUCTION}

The basic concept of the model category, introduced by Quillen [25], provides an extremely useful tool for developing axiomatic homotopy theory in very general situations (see, for instance, [21], [22], [19], [3]). Recall that a model category structure can be introduced into a category by specifying three classes of morphisms, called fibrations, cofibration and weak equivalences, which satisfy certain axioms. By passing to the "homotopy category" $\mathcal{H o}(\mathcal{C})$ of a model category $\mathcal{C}$ one formally inverts weak equivalences (if both domain and range are nice - cofibrant and fibrant simultaneously). In other words, in the quotient category $\mathcal{H o}(\mathcal{C})$ weak equivalences become homotopy equivalences. This fundamental fact manifests itself in different ways in particular situations. Even though the axioms of model categories are verifiable in a wide variety [14], [1], [20] of situations, they are reminiscent of well-known properties of homotopies for topological spaces. Obviously the category TOP of topological spaces itself possesses a model category structure (see, for instance, [14]).

Theorem A. The category TOP of topological spaces admits a model category structure where a map $f$ is a weak equivalence if $f$ is a weak homotopy

1991 Mathematics Subject Classification. Primary: 55U40; Secondary: 55U35, 18 D15.

Key words and phrases. model category, $[L]$-homotopy, $[L]$-complex.

The first author was partially supported by NSERC research grant. 
equivalence, i.e. for each $k=0,1, \ldots$ and each $x \in X$ the induced map $\pi_{k}(X, x) \rightarrow \pi_{k}(Y, f(x))$ is an isomorphism.

It should be noted that there exists [26] a model category structure on TOP whose weak equivalences are just standard homotopy equivalences. These two structures are essentially different although the weak equivalences between CWcomplexes in the corresponding homotopy categories become invertible, i.e. coincide with the ordinary homotopy equivalences. Clearly, for the latter structure this fact is true by definition (for any spaces). As for the former, the corresponding fact simply restates the following well known theorem of J.H.C.Whitehead $[28,29]$ (note that $C W$-complexes are cofibrant and fibrant in the first structure).

Theorem B. A map between $C W$-complexes (or, more generally, ANE-spaces) is a homotopy equivalence if and only if it induces isomorphisms of all homotopy groups.

There is one more type of model category structures on TOP closely related to the ordinary homotopies. Consider, for each $n=0,1, \ldots$, Whitehead's $n$ types [30] based on the concept of $n$-homotopy introduced in [17]. Algebraic models for $n$-types (the so called $c a t^{n}$-groups) were found in [24]. Approximately at the same time $n$-homotopies (and subsequently even $n$-shapes [7], [6]) begun to play a substantial role in the revitalized theory of Menger manifolds [2], [5] (see [8] for a discussion of categorical connections between $n$-homotopies and homotopies via the theories of manifolds modeled on Menger and Hilbert cubes respectively). The following theorem has been proved in [16].

Theorem C. Let $n=0,1, \ldots$ The category TOP of topological spaces admits a model category structure where a map $f: X \rightarrow Y$ is a weak equivalence if it is a weak n-homotopy equivalence, i.e. for each $k=0,1, \ldots, n$ and each $x \in X$ the induced map $\pi_{k}(X, x) \rightarrow \pi_{k}(Y, f(x))$ is an isomorphism.

The corresponding homotopy category $\mathcal{H} o_{n}(\mathbf{T O P})$ is a model category for the above mentioned notion of $n$-type. It is essential to note that the invertibility of weak equivalences in $\mathcal{H} o_{n}(\mathbf{T O P})$, in analogy with Theorem B, restates another well-known result of Whitehead [28].

Theorem D. A map between at most n-dimensional $C W$-complexes (or, more generally, at most $n$-dimensional $\mathrm{LC}^{n-1}$-spaces) is an n-homotopy equivalence if and only if it induces isomorphisms of the $k$-th homotopy groups for each $k \leq n-1$.

Below, for each finite CW-complex $L$ we consider the concept of $[L]$-homotopy introduced in [4]. We also present detailed description of $L$-homotopy groups also introduced in [4]. Our main result is as follows. 
Theorem 4.7. Let $L$ be a finite $C W$-complex. The category TOP of topological spaces admits a model category structure where a map $f: X \rightarrow Y$ is a weak equivalence if it is a weak $[L]$-homotopy equivalence, i.e. for each $n=0,1, \ldots$ and each $x \in X$ the induced map $\pi_{n}^{[L]}(X, x) \rightarrow \pi_{n}^{[L]}(Y, f(x))$ of $[L]$-homotopy groups is an isomorphism.

As above we conclude that weak equivalences become invertible in the corresponding homotopy category $\mathcal{H}_{[L]}(\mathbf{T O P})$. This proves the earlier announced result from [4, Theorem 2.9].

Corollary 4.8. A map between $[L]$-complexes is an $[L]$-homotopy equivalence if and only if it induces isomorphisms of all $[L]$-homotopy groups.

This result also extends both theorems $\mathrm{B}$ and $\mathrm{D}$. The first is obtained by letting $L=\{$ point $\}$ and the other by assuming $L=S^{n}$.

The concept of $[L]$-homotopy differs from the notions of homotopy or $n$ homotopy (see comment right before Definition 3.1) and it seems very interesting to develop this theory further as well as to consider corresponding homology and cohomology theories. There are some indications that such theories could be better designed for investigation of particular geometric constructions arising within the extension theory. Conversely, particular methods used in developing the theory of extension dimension (which led to $[L]$-homotopies) could be useful in building dimension theory in particular model categories.

The paper is organized as follows. In Section 2 we collect necessary definitions related to model categories and extension types of complexes. Section 3 discusses the concept of $[L]$-homotopy, introduced earlier by the first author. In particular, we define $[L]$-homotopy groups. Finally, in Section 4 , we prove our main result (Theorem 4.7) which provides an explicit description of a model category structure of TOP whose weak equivalences are precisely maps inducing isomorphisms of all $[L]$-homotopy groups.

The authors are grateful to the referee whose comments and suggestions led to a substantial improvement of the original exposition.

\section{Preliminaries}

In this section we present relevant definitions and results regarding model categories and extension dimension.

2.1. Model categories. We begin with the Quillen's concept of the model category.

Definition 2.1. A model category is a category $\mathcal{C}$ with three distinguished classes of maps:

(i) weak equivalences,

(ii) fibrations, 
(iii) cofibrations,

each of which is closed under compositions and contains all identity maps. A map which is both a fibration (respectively, cofibration) and a weak equivalence is called an acyclic fibration (respectively, acyclic cofibration). We require the following axioms:

(MC1) Finite limits and colimits exist in $\mathcal{C}$.

(MC2) If $f$ and $g$ are maps in $\mathcal{C}$ such that $g f$ is defined and if two of the three maps $f, g, g f$ are weak equivalences, then so is the third.

(MC3) If $f$ is a retract of $g$ and $g$ is a fibration, cofibration or a weak equivalence, then so is $f$.

(MC4) Given a commutative diagram

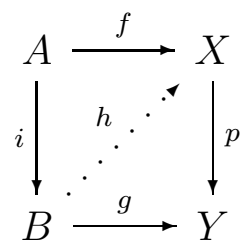

of unbroken arrows, a lift (the broken arrow) exists in either of the following two situations: (i) $i$ is a cofibration and $p$ is an acyclic fibration, or (ii) $i$ is an acyclic cofibration and $p$ is a fibration.

(MC5) Any map $f$ can be factored in two ways: (i) $f=p i$, where $i$ is a cofibration and $p$ is an acyclic fibration, and (ii) $f=q j$, where $j$ is an acyclic cofibration and $q$ is a fibration.

If $\mathcal{C}$ is a model category, then it has an initial object and a terminal object (the first being the colimit and the second being the limit of the empty diagram). An object in $\mathcal{C}$ is called cofibrant if the map from the initial object to it is a cofibration. Similarly an object in $\mathcal{C}$ is called fibrant if the map from it to the terminal object is a fibration. $\mathcal{C}_{c f}$ denotes the full subcategory of $\mathcal{C}$ consisting of objects which are simultaneously cofibrant and fibrant.

The following important observation is well-known ([14, Lemma 4.24], [20, Proposition 1.2.8]).

Proposition 2.2. Suppose $\mathcal{C}$ is a model category. Then a map of $\mathcal{C}_{c f}$ is a weak equivalence if and only if it is a homotopy equivalence.

2.2. Extension Types and Extension Dimension. For spaces $X$ and $L$, the notation $L \in \mathrm{A}(\mathrm{N}) \mathrm{E}(X)$ means that every map $f: A \rightarrow L$, defined on a closed subspace $A$ of $X$, admits an extension $\tilde{f}: X \rightarrow L$ (respectively, $\tilde{f}: G \rightarrow L$ ) over $X$ (respectively, over a neighborhood $G$ of $A$ in $X$ ).

This notation allows us to define a preorder relation $\leq$ on the class of $\mathrm{CW}$ complexes. Following [9], we say that $L \leq K$ if for each space $X$ the condition $L \in \mathrm{AE}(X)$ implies the condition $K \in \mathrm{AE}(X)$. The preorder relation $\leq$ naturally generates the equivalence relation: $L \sim K$ iff $L \leq K$ and $K \leq L$. We 
denote by $[L]$ the equivalence class of $L$. These equivalence classes of complexes are called extension types. The above defined relation $\leq$ creates a partial order in the class of extension types. This partial order will still be denoted by $\leq$. Note that under these definitions the class of all extension types has both maximal and minimal elements. The minimal element is the extension type of the 0-dimensional sphere $S^{0}$ (i.e. the two-point discrete space) and the maximal element is obviously the extension type of the one-point space $\{*\}$ (or, equivalently, of any contractible complex).

Example 2.3. The following observations express some basic properties of the above order.

(i) $\min \{[L],[K]\}=[L \vee K]$, here $L \vee K$ denotes the bouquet of complexes $K$ and $L$.

(ii) $\left[S^{0}\right]=[L] \Longleftrightarrow L$ is not connected.

(iii) $\left[S^{1}\right] \leq[L] \Longleftrightarrow L$ is connected.

(iv) It follows from the above two observations that there is no complex $L$ such that $\left[S^{0}\right]<[L]<\left[S^{1}\right]$.

(v) Clearly, $S^{n} \in \operatorname{AE}(X) \Longleftrightarrow \operatorname{dim} X \leq n$.

(vi) Similarly, $K(G, n) \in \mathrm{AE}(X) \Longleftrightarrow \operatorname{dim}_{G} X \leq n$. Here $\operatorname{dim}_{G} X$ stands for the cohomological dimension of $X$ with coefficients in an abelian group $G$ and $K(G, n)$ denotes the corresponding Eilenberg-MacLane complex, i.e. a complex satisfying the following conditions: $\pi_{n}(K(G, n))=G$ and $\pi_{k}(K(G, n))=0$ for each $k \neq n$.

(vii) Obviously $\left[S^{n}\right] \leq[K(\mathbb{Z}, n)]$, but $\left[S^{n}\right] \neq[K(\mathbb{Z}, n)]$. The last part follows from [10] (for $n=3$ ) and [15] (for $n=2$ ).

(viii) $\left[S^{n}\right]<\left[M\left(\mathbb{Z}_{2}, n+1\right) \vee S^{n+1}\right]<\left[S^{n+1}\right]$, where $M\left(\mathbb{Z}_{2}, n+1\right)$ is the corresponding Moore complex, $n \geq 1$.

(ix) The extension type $\left[\mathbb{R} P^{2}\right]$ of the projective plane is not comparable with $\left[S^{n}\right]$ for any $n \geq 2$ (see $\left.[12]\right)$.

The Homotopy Extension Theorem implies the following trivial observation.

Proposition 2.4. If $L$ and $K$ are homotopy equivalent complexes, then $[L]=$ $[K]$.

Observe that $\left[S^{n} \vee S^{n+1}\right]=\left[S^{n}\right]$ which shows that homotopy inequivalent complexes might have the same extension type.

The following notion is introduced by A. Dranishnikov (see [9] and [11]). The extension dimension of a Tychonov space $X$ is less than or equal to $[L]$ (briefly, $\mathrm{e}-\operatorname{dim}(X) \leq[L])$ if $X \in A E(L)$. More precisely

$$
\mathrm{e}-\operatorname{dim}(X)=\min \{[L]: L \in \operatorname{AE}(X)\} .
$$




\section{3. $[L]$-HOMOTOPIES AND $[L]$-COMPLEXES}

Throughout this section $L$ stands for a finite CW-complex. Let $A$ be a subspace of a Polish space $X$ and let $f_{0}, f_{1}: X \rightarrow Y$ be two maps such that $f_{0}(x)=f_{1}(x)$ for each $x \in A$. Then $f_{0}$ and $f_{1}$ are said to be $[L]$-homotopic relative to $A$ (notation: $f_{0} \stackrel{[L]}{\simeq} f_{1}$ rel $A$ ) if for any map $h: Z \rightarrow X \times[0,1]$ where $Z$ is a Polish space of extension dimension $\mathrm{e}-\operatorname{dim} Z \leq[L]$, there exists a map $H: Z \rightarrow Y$ such that

$$
H(z)=\left\{\begin{array}{l}
h\left(f_{0}(z)\right), \text { if } z \in h^{-1}(X \times\{0\} \cup A \times[0,1]), \\
h\left(f_{1}(z)\right), \text { if } z \in h^{-1}(X \times\{1\}) .
\end{array}\right.
$$

The following diagram illustrates the situation:

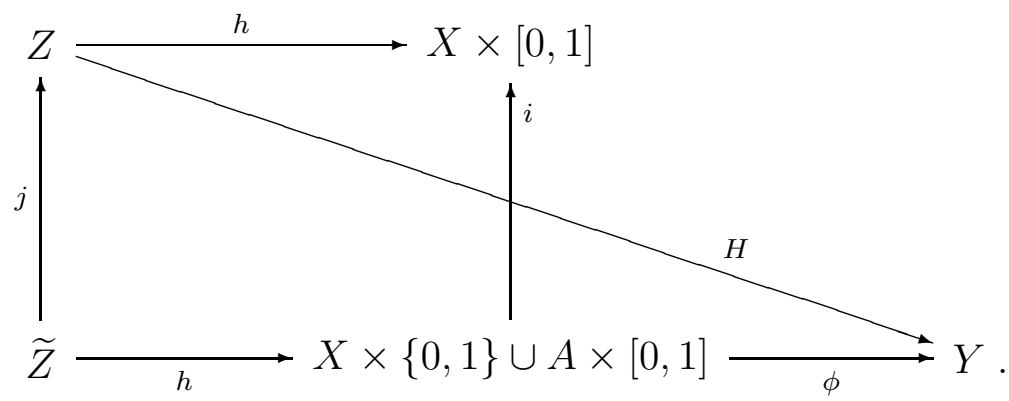

Here $\widetilde{Z}=h^{-1}(X \times\{0,1\} \cup A \times[0,1])$ and

$$
\phi(x)=\left\{\begin{array}{l}
f_{0}(x), \text { if } x \in X \times\{0\} \cup A \times[0,1], \\
f_{1}(x), \text { if } x \in X \times\{1\} .
\end{array}\right.
$$

If $A=\emptyset$, then we say that $f_{0}$ and $f_{1}$ are $[L]$-homotopic (notation: $f_{0} \stackrel{[L]}{\simeq} f_{1}$, see [4, Definition 2.9]).

It is easy to verify [4, Proposition 2.7] that extension dimension of a locally compact polyhedron is identical with its standard dimension. However the concept of $[L]$-homotopy differs from the classical concepts of homotopy or $n$-homotopy even for maps between finite polyhedra - this can be easily understood analyzing the identity map of the complex indicated in example 2.3(viii).

The class of spaces with respect to which $[L]$-homotopies behave well is identified in the following definition.

Definition 3.1 (Definition 2.2, [4]). We say that a space $X$ is an absolute (neighborhood) extensor modulo $L$, or shortly that $X$ is an $\mathrm{ANE}([L])$-space (notation: $X \in \operatorname{ANE}([L]))$ if $X \in \operatorname{ANE}(Y)$ for each space $Y$ with $\mathrm{e}-\operatorname{dim}(Y) \leq[L]$. 
Some of the basic properties of ordinary homotopies have their analogs for $[L]$-homotopies (see [4] for details). Here is an analog of the standard homotopy extension theorem [4, Proposition 2.28].

Proposition 3.2. Let $L$ be a finitely dominated complex and $X$ be a Polish $\operatorname{ANE}([L])$-space. Suppose that $A$ is closed in a space $B$ with $e-\operatorname{dim}(B) \leq[L]$. If maps $f, g: A \rightarrow X$ are $[L]$-homotopic and $f$ admits an extension $F: B \rightarrow X$, then $g$ also admits an extension $G: B \rightarrow X$, and it may be assumed that $F \stackrel{[L]}{\simeq} G$.

We also recall the following statement [4, Proposition 2.26].

Proposition 3.3. Let $L$ be a finitely dominated complex and $X$ be a Polish $\operatorname{ANE}([L])$-space $X$. Then there exists an open cover $\mathcal{U} \in \operatorname{cov}(X)$ such that any two $\mathcal{U}$-close maps of any space into $X$ are $[L]$-homotopic.

The class of approximately $[L]$-soft maps plays an important role in the $[L]$ homotopy theory. Between locally nice spaces of extension dimension not exceeding $[L]$, such maps provide basic examples of $[L]$-homotopy equivalences.

Definition 3.4 (Definition 2.6, [4]). A map $f: X \rightarrow Y$ is said to be approximately $[L]$-soft, if for each space $B$ with e $-\operatorname{dim}(B) \leq[L]$, for each closed subset $A$ of it, for an open cover $\mathcal{U} \in \operatorname{cov}(Y)$, and for any two maps $g: A \rightarrow X$ and $h: B \rightarrow Y$ such that $f g=h / A$, there is a map $k: B \rightarrow X$ satisfying the conditions $k / A=g$ and the composition $f k$ is $\mathcal{U}$-close to $h$.

Important examples of approximately $L$-soft maps between polyhedra are those whose non-trivial fibers are topological (or, more generally, homotopical) copies of the complex $L$.

Proposition 3.5. Let $p: X \rightarrow Y$ be an approximately $[L]$-soft map between Polish spaces. Let also $f_{1}, f_{2}: A \rightarrow X$ be two maps, defined on a Polish space A, such that

(a) $f_{1}\left(a_{0}\right)=f_{2}\left(a_{0}\right)$ for some point $a_{0} \in A$;

(b) $p \circ f_{0} \stackrel{[L]}{\simeq} p \circ f_{1} \operatorname{rel} a_{0}$.

Then $f_{0} \stackrel{[L]}{\simeq} f_{1}$ rel $a_{0}$.

Proof. Consider the map $\phi: A \times\{0,1\} \cup\left\{a_{0}\right\} \times I \rightarrow Y$, defined by letting

$$
\phi(a, t)=\left\{\begin{array}{l}
p\left(f_{t}(a)\right), \text { if } a \in A \text { and } t=0,1 \\
p\left(f_{0}\left(a_{0}\right)\right), \text { if } a=a_{0} \text { and } t \in I
\end{array}\right.
$$

Let also $h: Z \rightarrow A \times I$ be an $[L]$-invertible map such that e $-\operatorname{dim} Z \leq[L]$ and $\widetilde{Z}=h^{-1}\left(A \times\{0,1\} \cup\left\{a_{0}\right\} \times I\right)$. By (b), there exists a map $H: Z \rightarrow Y$ which extends the composition $\phi \circ h \mid \widetilde{Z}: \widetilde{Z} \rightarrow Y$.

Next consider the map $\varphi: A \times\{0,1\} \cup\left\{a_{0}\right\} \times I \rightarrow X$ 


$$
\varphi(a, t)=\left\{\begin{array}{l}
f_{t}(a), \text { if } a \in A \text { and } t=0,1 \\
f_{0}\left(a_{0}\right), \text { if } a=a_{0} \text { and } t \in[0,1]
\end{array}\right.
$$

which, according to (a), is well defined.

It is easy to see that the following diagram of unbroken arrows commutes (here $i$ and $j$ denote the corresponding inclusion maps).

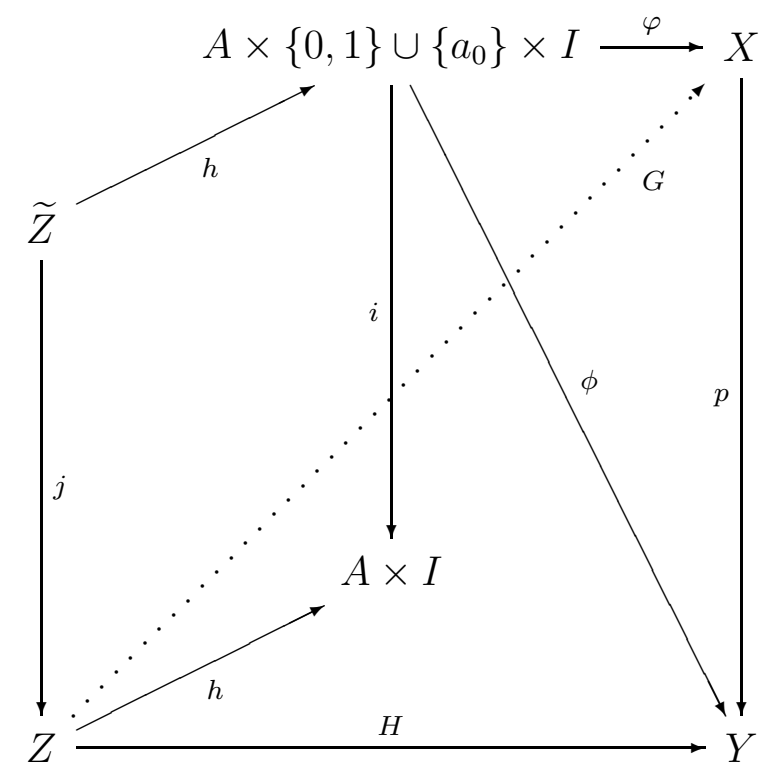

In particular, $p \circ \varphi \circ h|\widetilde{Z}=\phi \circ h| \widetilde{Z}=H \mid \widetilde{Z}$. Finally, since $p$ is approximately $[L]$-soft and since e $-\operatorname{dim} Z \leq\{L]$ it follows that there exists a map $G: Z \rightarrow X$ (the broken arrow in the above diagram) such that $G|\widetilde{Z}=\varphi \circ h| \widetilde{Z}$ (notice also that above diagram is not commutative if $G$ is included in it, however, it can be made approximately commutative). This proves that $f_{0} \stackrel{[L]}{\simeq} f_{1} \operatorname{rel} a_{0}$.

3.1. $[L]$-complexes. It is well known that one of the primary goals of introducing the concept of $C W$-complexes was the possibility of developing a satisfactory homotopy theory. The class of spaces which is specifically designed for the needs of $[L]$-homotopy theory can be defined similarly. First we need the following resolution theorem (statements (i)-(iii) below are contained in [Proposition 2.23][4]).

Proposition 3.6 (Proposition 2.23, [4]). Let $L$ be a finite complex and $X$ be a locally finite polyhedron. Then there exist a locally compact metrizable space $\mu_{X}^{[L]}$ and an $[L]$-invertible and approximately $[L]$-soft proper map $f_{X}^{[L]}: \mu_{X}^{[L]} \rightarrow X$ satisfying the following conditions:

(i) $\mu_{X}^{[L]} \in \operatorname{ANE}([L])$. 
(ii) $e-\operatorname{dim}\left(\mu_{X}^{[L]}\right)=[L]$.

(iii) For any map $f: B \rightarrow \mu_{X}^{[L]}$, where $B$ is a compact space with $e-\operatorname{dim}(B) \leq$ $[L]$, and for any open cover $\mathcal{U} \in \operatorname{cov}\left(\mu_{X}^{[L]}\right)$ there is an embedding $g: B \rightarrow$ $\mu_{X}^{[L]}$ which is $\mathcal{U}$-close to $f$ and such that $f_{X}^{[L]} \circ g=f_{X}^{[L]} \circ f$.

(iv) If, in addition, $\tau$ is a triangulation of $X$, then one can assume that for any subpolyhedron $Y$ of $X$ (with respect to $\tau$ ), the inverse image $\left(f_{X}^{[L]}\right)^{-1}(Y)$ is also a locally compact $\mathrm{ANE}([L])$-space and the restriction $f_{X}^{[L]} \mid\left(f_{X}^{[L]}\right)^{-1}(Y):\left(f_{X}^{[L]}\right)^{-1}(Y) \rightarrow Y$ is also approximately $[L]$-soft.

Proof. Proof of this statement, with minor adjustments, follows Dranishnikov's construction [12]. Lemmas 2.6 and 2.7 from [4] (see also [12, Lemmas 2.2 and 2.3]) allow us to construct inductively an inverse sequence $\mathcal{S}=\left\{X_{n}, p_{n}^{n+1}\right\}$, consisting of locally compact polyhedra $X_{n}$ (with certain triangulations whose meshes converge to zero) and $[L]$-invertible, approximately $[L]$-soft, proper, simplicial bonding maps $p_{n}^{n+1}: X_{n+1} \rightarrow X_{n}$ so that $X_{1}$ is the given polyhedron $X$ considered with the given triangulation $\tau$. As in [12, Lemma 2.3], we may assume that $\mathcal{S}$ is $L$-resolvable inverse sequence. We let $\mu_{X}^{[L]}=\lim \mathcal{S}$ and let the map $f_{X}^{[L]}: \mu_{X}^{[L]} \rightarrow X$ be the limit projection $p_{\infty}: \lim \mathcal{S} \rightarrow X_{1}$ of the given spectrum. As in the proof of [12, Theorem 2.4], we have e $-\operatorname{dim}\left(\mu_{X}^{[L]}\right) \leq[L]$ (Property (ii)). Property (i) follows from [4, Proposition 2.22]. Property (iv) is satisfied by construction. If, during the inductive construction, we define $X_{n+1}$ insuring that the bonding map $p_{n}^{n+1}: X_{n+1} \rightarrow X_{n}$ factors through the projection $X_{n} \times[0,1] \rightarrow X_{n}$, then one can easily verify that property (iii) would be also satisfied.

Below, for each $n \geq 0$, we consider at most $[L]$-dimensional compact $\mathrm{AE}([L])$ spaces $D_{[L]}^{n}$ which admit ([L]-invertible) approximately $[L]$-soft maps onto $n$ dimensional disk $D^{n}$. Actually Proposition 3.6(iv) allows us to consider pairs $\left(S_{[L]}^{n}, D_{[L]}^{n+1}\right)$ as approximately $[L]$-soft preimages of the standard pairs $\left(S^{n}, D^{n+1}\right)$ consisting of the $(n+1)$-dimensional disk and its boundary $\partial D^{n+1}=S^{n}, n \geq 0$. We say that $D_{[L]}^{n}$ is an $n$ - $[L]-$ disk and that $S_{[L]}^{n}$ is an $n$ - $[L]$-sphere. Note that generally speaking for any given $n$ there are many $n$ - $[L]$-disks and $n$ - $[L]$-spheres. Clearly all $n$ - $[L]$-disks, as $\mathrm{AE}([L])$-compacta, are $[L]$-homotopy equivalent to the one point spaces. Proposition 3.5 shows that all $n$ - $[L]$-spheres are also $[L]$-homotopy equivalent.

The following concept has, in fact, been introduced in [4, Section 2.6.2].

Definition 3.7. We say that a space $X$ of extension dimension e- $\operatorname{dim} X \leq[L]$ is a (finite) $[L]$-polyhedron if it admits a proper approximately $[L]$-soft map $f: X \rightarrow Y$ onto a locally finite polyhedron $Y$ such that $f$ possesses property (iv) of Proposition 3.6 for some (finite) triangulation $\tau$ of $Y$. By $[L]$-complexes 
we understand spaces $X$ with $\mathrm{e}-\operatorname{dim} X \leq[L]$ that are $[L]$-homotopy equivalent to $[L]$-polyhedra.

The above definition guarantees that any $[L]$-polyhedron can be constructed by attaching "cells" (i.e. $[L]$-disks) along their "boundaries" (i.e. $[L]$-spheres) by means of inclusion maps. Therefore a wide variety of properties of $[L]$-polyhedra can be obtained by straightforward modifications of standard constructions and proofs (see, for instance, [18], [27]).

Next, suppose that $X$ is an $[L]$-polyhedron and $f: X \rightarrow Y$ is a corresponding approximately $[L]$-soft mapping onto a locally finite polyhedron $Y$. Let $Y^{(n)}$ denote the $n$-skeleton of $Y$. Then for any $y \in Y^{(0)}$ we have $f^{-1}(y) \in A E([L])$. Denote $X^{(n)}=f^{-1}\left(Y^{(n)}\right)$. We call $X^{(n)}$ a $n$ - $[L]$-skeleton of $X$. Clearly, $X^{(n)}$ is closed in $X$ for any $n$. Since $Y$ is locally finite for any point $x \in X$ there exists an open (in $X$ ) neighbourhood $O x$ such that $O x \subset X^{(n)}$ for some $n$. This implies that a subset $F$ of $X$ is closed if and only if $F \cap X^{(n)}$ is closed for any $n$.

Every Hilbert cube manifold, according to the corresponding triangulation theorem, is topologically homeomorphic to the product $K \times \mathbb{Q}$ of a locally compact polyhedron $K$ and the Hilbert cube $\mathbb{Q}$. Since the projection $K \times \mathbb{Q} \rightarrow$ $K$ is a proper (approximately) soft map, it follows that every Hilbert cube manifold is a $[\{*\}]$-polyhedron. It is important to note however that every $[\{*\}]$-polyhedron is homotopically equivalent to a standard polyhedron.

$\mu^{n}$-manifolds (i.e. spaces locally homeomorphic to the $n$-dimensional universal Menger compactum $\mu^{n}$ ) can also be "triangulated" in certain non-standard sense (see a comprehensive discussion of related matters in [13], [5, Chapter $4]$ ). In particular, they admit proper approximately $\left[S^{n}\right]$-soft maps onto standard polyhedra and consequently are $\left[S^{n}\right]$-polyhedra in our sense. They are $\left[S^{n}\right]$-homotopy equivalent to standard $n$-dimensional polyhedra.

3.2. $[L]$-homotopy groups. Let $S^{n}$ denote a unit $n$-sphere. Fix a point $s \in$ $S^{n}$. For each $n \geq 0$ we consider an $n$ - $[L]-$ sphere, i.e. a compactum of extension dimension at most $[L]$ which admits an approximately $[L]$-soft mapping onto $S^{n}$.

Let $\left(X, x_{0}\right)$ be a pointed space. Let $S_{[L]}^{n}$ be a $n$-[L]-sphere, $n \geq 1$, and $f: S_{[L]}^{n} \rightarrow S^{n}$ be an approximately $[L]$-soft mapping. Fix a point $s_{[L]} \in$ $f^{-1}(s)$ and consider the set $\pi_{n}^{[L]}\left(X, x_{0}\right)=\left[\left(S_{[L]}^{n}, s_{[L]}\right),\left(X, x_{0}\right)\right]_{[L]}$ of relative $[L]-$ homotopy classes of maps of pointed spaces.

Next, we show that this set does not depend on the choice of $[L]$-sphere. Consider another $n$ - $[L]$-sphere $Q_{[L]}^{n}$ and let $g: Q_{[L]}^{n} \rightarrow S^{n}$ be the corresponding approximately $[L]$-soft mapping. Choose a point $q_{[L]} \in g^{-1}(s)$ and let $\tilde{\pi}_{n}^{[L]}\left(X, x_{0}\right)=\left[\left(Q_{[L]}^{n}, q_{[L]}\right),\left(X, x_{0}\right)\right]_{[L]}$. 
Since $S^{n}$ is an ANE-compactum there exists an open cover $\mathcal{U} \in \operatorname{cov}\left(S^{n}\right)$ such that any two $\mathcal{U}$-close maps (defined on any compactum) are homotopic as maps into $S^{n}$ (this is Proposition 3.3 with $L=\{*\}$ ).

Now consider the following commutative diagram (consisting of unbroken arrows)

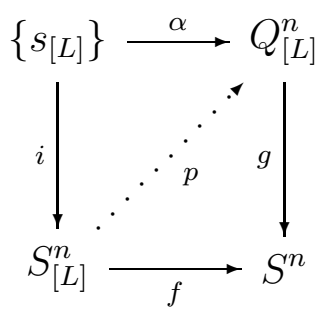

where $\alpha\left(s_{[L]}\right)=q_{[L]}$.

Since $g$ is approximately $[L]$-soft, there exists a mapping $p: S_{[L]}^{n} \rightarrow Q_{[L]}^{n}$ (the broken arrow in the above diagram) such that $p\left(s_{[L]}\right)=q_{[L]}($ i.e. $p \circ i=\alpha)$ and $g \circ p$ is $\mathcal{U}$-close to $f$. Similarly, there exists a mapping $q: Q_{[L]}^{n} \rightarrow S_{[L]}^{n}$ such that $q\left(q_{[L]}\right)=s_{[L]}$ and $f \circ q$ is $\mathcal{U}$-close to $g$. Choice of the cover $\mathcal{U}$ guarantees that $g \circ p \simeq f$ and $f \circ q \simeq g$. We may assume without loss of generality that these are homotopies relative to the given points $s_{[L]}$ and $q_{[L]}$.

Next note that

$$
g \circ p \circ q \simeq f \circ q \operatorname{rel} q_{[L]} \simeq g \operatorname{rel} q_{[L]}
$$

and

$$
f \circ q \circ p \simeq g \circ p \operatorname{rel} s_{[L]} \simeq f \operatorname{rel} s_{[L]}
$$

According to Proposition 3.5, qop $\stackrel{[L]}{\simeq} \operatorname{id}_{Q_{[L]}^{n}} \operatorname{rel} q_{[L]}$ and $p \circ q \stackrel{[L]}{\simeq} \operatorname{id}_{S_{[L]}^{n}} \operatorname{rel} s_{[L]}$. We shall refer to mappings constructed as described above as $[L]$-homotopy equivalences of canonical type. Observe, that any two $[L]$-homotopy equivalences of canonical type are $[L]$-homotopic.

Define a mapping $\phi: \pi_{n}^{[L]}\left(X, x_{0}\right) \rightarrow \widetilde{\pi}_{n}^{[L]}\left(X, x_{0}\right)$ as follows. Consider an element $\alpha \in \pi_{n}^{[L]}\left(X, x_{0}\right)$ and let $a:\left(S_{[L]}^{n}, s_{[L]}\right) \rightarrow\left(X, x_{0}\right)$ be its representative. We let $\phi(\alpha)=\beta$, where $\beta$ is a relative $[L]$-homotopy class of the composition $a \circ q:\left(Q_{[L]}^{n}, q_{[L]}\right) \rightarrow\left(X, x_{0}\right)$. Similarly, we define a mapping $\psi: \tilde{\pi}_{n}^{[L]}\left(X, x_{0}\right) \rightarrow$ $\pi_{n}^{[L]}\left(X, x_{0}\right)$ using the mapping $p$. It is easy to check that $\phi$ and $\psi$ are welldefined. Clearly they are inverses to each other. Thus, there exists a bijection 
between the sets $\pi_{n}^{[L]}\left(X, x_{0}\right)$ and $\tilde{\pi}_{n}^{[L]}\left(X, x_{0}\right)$. Therefore, the set $\pi_{n}^{[L]}\left(X, x_{0}\right)$ does not depend on the choice of $n$ - $[L]$-sphere. It is clear that bijections $\phi$ and $\psi$ do not depend on the choice of $[L]$-homotopy equivalences of canonical type.

Let $f:\left(X, x_{0}\right) \rightarrow\left(Y, y_{0}\right)$ be a mapping and $[f]_{[L]} \in\left[\left(X, x_{0}\right),\left(Y, y_{0}\right)\right]_{[L]}$ be its relative $[L]$-homotopy class. Then a natural map $\pi_{n}^{[L]}\left([f]_{[L]}\right): \pi_{n}^{[L]}\left(X, x_{0}\right) \rightarrow$ $\pi_{n}^{[L]}\left(Y, y_{0}\right)$ can be defined in a standard way.

Our next goal is to introduce a group structure on $\pi_{n}^{[L]}\left(X, x_{0}\right)$ such that natural maps $\pi_{n}^{[L]}\left([f]_{[L]}\right)$ are well-defined homomorphisms. Moreover, this structure will be defined so that bijections $\phi$ and $\psi$, generated by $[L]$-homotopy equivalences of canonical type, are group isomorphisms.

Let $\alpha$ and $\beta$ be two elements of the set $\pi_{n}^{[L]}\left(X, x_{0}\right)$ and $a, b:\left(S_{[L]}^{n}, s_{[L]}\right) \rightarrow$ $\left(X, x_{0}\right)$ be their representatives. Let $S_{+}^{n}$ and $S_{-}^{n}$ denote the upper and lower hemispheres, respectively, and $E$ denote an equator of $S^{n}$ containing the point $s$. Let $f: S_{[L]}^{n} \rightarrow S^{n}$ be an approximately $[L]$-soft mapping. Let $h: S^{n} \rightarrow S^{n} \vee S^{n}$ be the homotopy comultiplication defining the standard $H$-cogroup structure (see, for instance, [27, Definition 2.16]) of the sphere $S^{n}$. Let also $f_{-}: S_{[L]}^{n} \rightarrow S^{n}$ and $f_{+}: S_{[L]}^{n} \rightarrow S^{n}$ be two copies of the map $f$.

Since $f_{+}$is approximately $[L]$-soft there exists a mapping $\widetilde{a}: f^{-1}\left(S_{+}^{n}\right) \rightarrow S_{[L]}^{n}$ such that $\widetilde{a}\left(f^{-1}(E)\right)=s_{[L]}$ and the composition $f_{+} \circ \widetilde{a}$ is $\mathcal{U}$-close to the composition $\left.h \circ f\right|_{f^{-1}\left(S_{+}^{n}\right)}$. Similarly, there exists a mapping $\widetilde{b}: f^{-1}\left(S_{-}^{n}\right) \rightarrow S_{[L]}^{n}$ such that $\widetilde{b}\left(f^{-1}(E)\right)=s_{[L]}$ and the composition $f_{-} \circ \widetilde{b}$ is $\mathcal{U}$-close to the composition $\left.h \circ f\right|_{f^{-1}\left(S_{-}^{n}\right)}$. Here is the corresponding diagram (commutative up to $[L]$-homotopy):

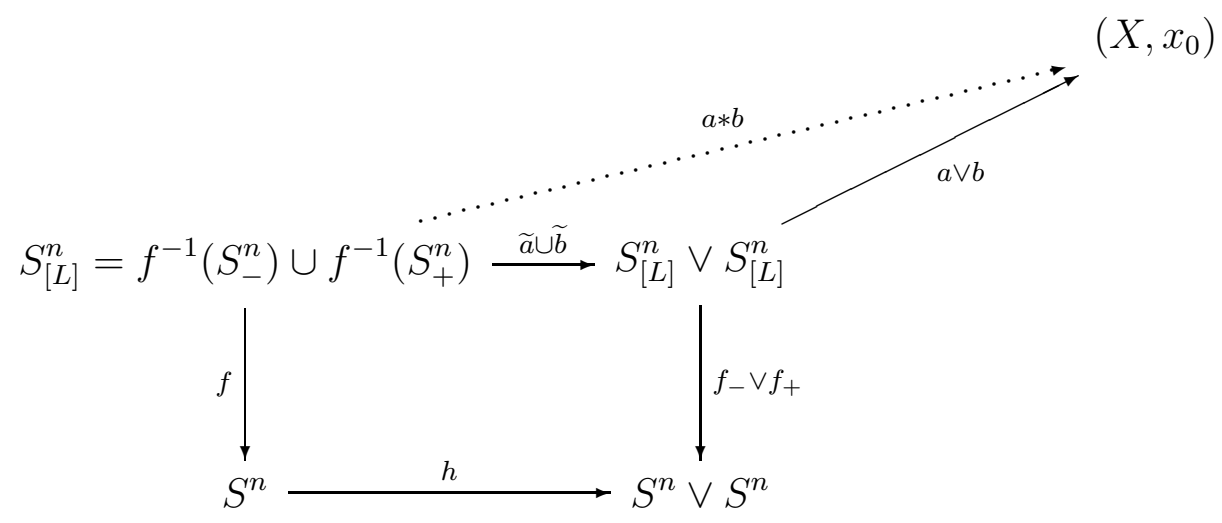


It is routine to check that the relative $[L]$-homotopy class $[a * b]_{[L]}$ of the composition $a * b=(\widetilde{a} \cup \widetilde{b}) \circ(a \vee b):\left(S_{[L]}^{n}, E^{\prime}\right) \rightarrow\left(X, x_{0}\right)$, which formally is defined by letting

$$
(a * b)(x)= \begin{cases}a(\widetilde{a}(x)), & \text { if } x \in f^{-1}\left(S_{+}^{n}\right) \\ b(\widetilde{b}(x)), & \text { if } x \in f^{-1}\left(S_{-}^{n}\right)\end{cases}
$$

does not depend on the choice of representatives $a$ and $b$ (and mappings $\widetilde{a}$ and $\widetilde{b})$. Now we can define the product of $\alpha$ and $\beta$ by letting $\alpha * \beta=[a * b]_{[L]}$.

The unit element in $\pi_{n}^{[L]}\left(X, x_{0}\right)$ is given by $\epsilon=[e]_{[L]}$ where $e$ is a constant mapping which sends $S_{[L]}^{n}$ to the point $x_{0}$.

Finally, given an element $\alpha \in \pi_{n}^{[L]}\left(X, x_{0}\right)$ we define its inverse $\alpha^{-1} \in \pi_{n}^{[L]}\left(X, x_{0}\right)$ with respect to the operation $*$ as follows. Let $a:\left(S_{[L]}^{n}, s_{[L]}\right) \rightarrow\left(X, x_{0}\right)$ be a representative of $\alpha$. Let $g: S^{n} \rightarrow S^{n}$ be the mapping such that $g\left(x_{1}, \ldots, x_{n+1}\right)=$ $\left(x_{1}, \ldots,-x_{n+1}\right)$, which fixes the equator $E$. Since $f$ is approximately $[L]$-soft there exists a mapping $\widetilde{g}: S_{[L]}^{n} \rightarrow S_{[L]}^{n}$ such that $\widetilde{g}\left(s_{[L]}\right)=s_{[L]}$ and composition $f \circ \widetilde{g}$ is $\mathcal{U}$-close to the composition $g \circ f$. Here is the diagram (as before commutative up to $[L]$-homotopy):

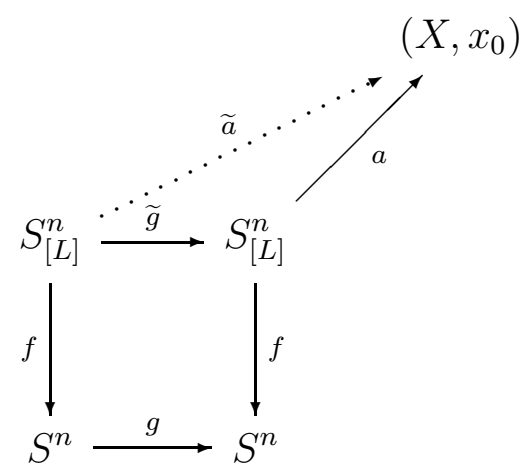

The $[L]$-homotopy class of the composition $\widetilde{a}=a \circ \widetilde{g}:\left(S_{[L]}^{n}, s_{[L]}\right) \rightarrow\left(X, x_{0}\right)$ does not depend on the choice of representative $a$ and of the mapping $\widetilde{g}$. This allows us to define $\alpha^{-1}=[a \circ \widetilde{g}]_{[L]}$. It only remains to note that $\alpha^{-1} \alpha=\alpha \alpha^{-1}=\epsilon \epsilon$ $\pi_{n}^{[L]}\left(X, x_{0}\right)$ 


\section{Model Category structure on TOP Generated By a Finite COMPLEX}

In this section we prove our main results which states that the category TOP admits a model category structure whose weak equivalences are weak $[L]$-homotopy equivalences. We begin by introducing the needed classes of morphisms.

Definition 4.1. A map $f: X \rightarrow Y$ of spaces is called a weak $[L]$-homotopy equivalence if for each basepoint $x \in X$ the map $f_{*}: \pi_{n}^{[L]}(X, x) \rightarrow \pi_{n}^{[L]}(Y, f(x))$ is a bijection of pointed sets for $n=0$ and an isomorphism of groups for $n \geq 1$.

In order to define cofibrations and fibrations first recall that a map $i: A \rightarrow B$ has the left lifting property (LLP) with respect to a map $p: X \rightarrow Y$ and $p$ has the right lifting property (RLP) with respect to $i$ if, for every commutative square diagram of unbroken arrows

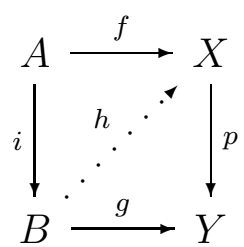

there exists a lift (the broken arrow) $h: B \rightarrow X$ such that $h \circ i=f$ and $p \circ h=g$.

Definition 4.2. Let $f: X \rightarrow Y$ be a map in TOP. We say that $f$ is

(i) ${ }_{L}$ a weak equivalence if it is a weak $[L]$-homotopy equivalence,

(ii) ${ }_{L}$ a fibration if it has the RLP with respect to inclusions of finite $[L]$-polyhedra $A \hookrightarrow B$ such that both $A$ and $B$ are $A E([L])$-spaces.

(iii) ${ }_{L}$ a cofibration if it has the LLP with respect to acyclic fibrations (see Definition 2.1).

Below we shall use the following fact, proof of which is trivial.

Proposition 4.3. Let $f: S_{[L]}^{n} \rightarrow X$ be a mapping of $n$-[L]-sphere to a topological space $X$. If $f$ is $[L]$-homotopic to a constant map then for any embedding of $S_{[L]}^{n}$ to a space $Z$ of extension dimension $\leq[L]$ the mapping $f$ can be extended to a mapping of $Z$ to $X$. Conversely, suppose that $S_{[L]}^{n}$ is a subspace of a space $Z$ of extension dimension $\leq[L]$ such that $Z \in A E([L])$ and $f$ admits an extension over $Z$. Then $f$ is $[L]$-homotopic to a constant map.

In order to verify axioms of model category we need to obtain a characterization of acyclic $[L]$-fibrations in terms of the RLP.

Lemma 4.4. An $[L]$-fibration $p: X \rightarrow Y$ is acyclic iff it has the $R L P$ with respect to any pair $S_{[L]}^{n} \subset D_{[L]}^{n+1}$, where $D_{[L]}^{n+1}$ is an $(n+1)-[L]$-disk and $S_{[L]}^{n}$ is a corresponding $n$ - $[L]$-sphere, $n=0,1, \ldots$. 
Proof. First let us prove the following claim.

Claim. Let $p: X \rightarrow Y$ be an acyclic $[L]$-fibration. Then there exist pairs of $[L]$-polyhedra $\left(S_{[L]}^{n}, D_{[L]}^{n+1}\right)$, where $D_{[L]}^{n+1}$ is an $(n+1)$ - $[L]$-disk and $S_{[L]}^{n}$ is the corresponding $n$ - $[L]$-sphere, $n=0,1, \ldots$, such that $p$ has the $R L P$ with respect to inclusions $S_{[L]}^{n} \subset D_{[L]}^{n+1}$.

Let $D_{r}^{n+1}$ and $S_{r}^{n}=\partial D_{r}^{n+1}$ denote centered at the origin $O$ (of the $(n+1)$ dimensional Euclidean space $\left.\mathbb{R}^{n+1}\right)(n+1)$-disk and $n$-sphere of radius $r$, respectively. Let $f: D \rightarrow D_{1}^{n+1}=D^{n+1}$ be an approximately $[L]$-soft mapping of a compactum $D$ having extension dimension $\leq[L]$ onto $D^{n+1}$. Put $S_{[L]}^{n}=f^{-1}\left(S_{1}^{n}\right), S_{[L]}^{n}(0)=f^{-1}\left(S_{1 / 2}^{n}\right), C_{[L]}^{n+1}=f^{-1}\left(D^{n+1} \backslash \operatorname{Int}\left(D_{1 / 2}^{n+1}\right)\right)$ and $D_{0}=$ $f^{-1}\left(D_{1 / 2}^{n+1}\right)$. Let $\tau$ denote a triangulation on $D^{n+1}$ such that $S_{1 / 2}^{n}, S_{1}^{n}, D_{1 / 2}^{n+1}$ and $D^{n+1} \backslash \operatorname{Int}\left(D_{1 / 2}^{n+1}\right)$ are subpolyhedra of $\tau$. According to Proposition 3.6, one can construct mapping $f$ so that restrictions of $f$ on preimages of subpolyhedra of $D^{n+1}$ with respect to $\tau$ are also approximately $[L]$-soft.

This implies, in particular, that $D_{0} \in A E([L]), S_{[L]}^{n} \in A N E([L])$ and that $C_{[L]}^{n+1}$ is an $(n+1)$ - $[L]-$ cylinder, i.e. $\operatorname{ANE}([\mathrm{L}])$-compactum of extension dimension $\leq[L]$ admitting an approximately $[L]$-soft mapping $f: C_{[L]}^{n+1} \rightarrow S^{n} \times I$, where $I=[0,1]$ denotes unit interval.

Let $D_{[L]}^{n+1}=D / D_{0}$ be a quotient space and $\phi: D \rightarrow D / D_{0}$ be a quotient mapping. Since $D \in A E([L])$ and $D_{0} \in A E([L])$ it follows that $D_{[L]}^{n+1} \in A E([L])$. Put $s_{[L]}=\phi\left(D_{0}\right)$. Define a mapping $g: D_{[L]}^{n+1} \rightarrow D^{n+1}$ as follows. We let $g\left(s_{[L]}\right)=O$ and for each point $x \in D_{[L]}^{n+1}$, distinct from $s_{[L]}$, we let $g(x)=$ $h \circ f(x)$, where $h: D^{n+1} \rightarrow D^{n+1}$ is a mapping which collapses $D_{1 / 2}^{n+1}$ to the point $O$. Let also $\tau^{\prime}$ denote a triangulation on $D^{n+1}$ obtained from $\tau$ by means of collapsing $D^{n+1}$ to point (and enlarging of the resulting cellular structure). It is easy to check that the mapping $g$ is approximately $[L]$-soft and restriction of $g$ on a preimage of any subpolyhedron of $D^{n+1}$ with respect to $\tau^{\prime}$ is also approximately $[L]$-soft. Therefore the constructed space $D_{[L]}^{n+1}$ is an $[L]$-polyhedron. Further in the proof of Claim we shall refer to the compacta constructed above simply as $[L]$-disk, corresponding $[L]$-sphere and $[L]$-cylinder, and denote them by $D_{[L]}^{n+1}, S_{[L]}^{n}$ and $C_{[L]}^{n+1}$, respectively. Notice also that $D_{[L]}^{n+1}$ is homeomorphic to a quotient space $C_{[L]}^{n+1} / S_{[L]}^{n}(0)$.

Let us show that the above constructed pair $\left(S_{[L]}^{n}, D_{[L]}^{n+1}\right)$ satisfies condition of the Claim. Consider two mappings $f: D_{[L]}^{n+1} \rightarrow Y$ and $G: S_{[L]}^{n} \rightarrow X$ such that $p \circ G=\left.f\right|_{S_{[L]}^{n}}$. Since $p$ is acyclic and $\left.f\right|_{S_{[L]}^{n}}$ represents trivial element of the group $\pi_{n}^{[L]}(Y)$, there exists a mapping $\bar{G}: D_{[L]}^{n+1} \rightarrow X$ extending $G$. 
Consider a compactum $\widehat{S_{[L]}^{n+1}}=\left(D_{[L]}^{n+1}\right)_{+} \cup\left(D_{[L]}^{n+1}\right)_{-}$obtained by gluing of two copies of $D_{[L]}^{n+1}$ along $S_{[L]}^{n}$. Clearly, $\widehat{S_{[L]}^{n+1}}$ admits an approximately $[L]-$ soft mapping onto $S^{n+1}$ and hence represents an $(n+1)-[L]$-sphere. Fix the point $s_{[L]} \in\left(D_{[L]}^{n+1}\right)_{-}$(see the construction of $D_{[L]}^{n+1}$ above). Define a mapping $h:\left(\widehat{S_{[L]}^{n+1}}, s_{[L]}\right) \rightarrow\left(Y, h\left(s_{[L]}\right)\right)$ as follows:

$$
h(x)= \begin{cases}f(x), & \text { if } x \in\left(D_{[L]}^{n+1}\right)_{+} \\ p \circ \bar{G}(x), & \text { if } x \in\left(D_{[L]}^{n+1}\right)_{-}\end{cases}
$$

Denote $y_{0}=h\left(s_{[L]}\right)$. Then $h$ represents an element $\alpha$ of the group $\pi_{n+1}^{[L]}\left(Y, y_{0}\right)$. Since $p$ is acyclic, there exists a mapping $H:\left(\widehat{S_{[L]}^{n+1}}, s_{[L]}\right) \rightarrow\left(X, x_{0}\right)$ where $x_{0} \in$ $p^{-1}\left(y_{0}\right)$ such that composition $p \circ H:\left(\widehat{S_{[L]}^{n+1}}, s_{[L]}\right) \rightarrow\left(Y, y_{0}\right)$ represents an element of the group $\pi_{n+1}^{[L]}\left(Y, y_{0}\right)$ which is $[L]$-homotopically inverse to $\alpha$.

Let $C_{[L]}^{n+1}$ be an $n$ - $[L]$-cylinder corresponding to $D_{[L]}^{n+1}$. Consider the space $\widehat{D_{[L]}^{n+1}}=D_{[L]}^{n+1} \cup C_{[L]}^{n+1}$ obtained by gluing of $D_{[L]}^{n+1}$ and $C_{[L]}^{n+1}$ along $S_{[L]}^{n}$ and the space $\widehat{S_{[L]}^{n+1}}=\left(\widehat{D_{[L]}^{n+1}}\right)+\cup\left(\widehat{D_{[L]}^{n+1}}\right)$ - obtained by gluing of two copies of $\widehat{D_{[L]}^{n+1}}$ along $S_{[L]}^{n}(0)$. Denote by $\phi$ the corresponding quotient mapping. It is easy to check that compactum $\widetilde{S_{[L]}^{n+1}}$ represents a $(n+1)$ - $[L]$-sphere. Let

$$
\widetilde{D_{[L]}^{n+1}}=\left(C_{[L]}^{n+1}\right)_{+} \cup_{\phi}\left(\widehat{D_{[L]}^{n+1}}\right)_{-} \subset \widetilde{S_{[L]}^{n+1}}
$$

Clearly, $\widetilde{D_{[L]}^{n+1}}$ is an $(n+1)$ - $[L]$-disk and an $[L]$-polyhedron. In particular, $\widetilde{D_{[L]}^{n+1}} \in$ $A E([L])$ Define a mapping

$$
\left.k: \widetilde{S_{[L]}^{n+1}}, S_{[L]}^{n}(0)\right) \rightarrow\left(Y, y_{0}\right)
$$

as follows:

$$
k(x)= \begin{cases}h(x), & \text { if } x \in\left(\widehat{D_{[L]}^{n+1}}\right)_{+} \backslash S_{[L]}^{n}(0) \\ p \circ H(x), & \text { if } x \in\left(\widehat{D_{[L]}^{n+1}}\right)_{-} \backslash S_{[L]}^{n}(0) \\ y_{0}, & \text { if } x \in S_{[L]}^{n}(0)\end{cases}
$$

Define a mapping $K:\left(\widetilde{D_{[L]}^{n+1}}\right) \rightarrow X$ such that $\left.k\right|_{\widetilde{D_{[L]}^{n+1}}}=p \circ K$ by letting

$$
K(x)= \begin{cases}\bar{G}(x), & \text { if } x \in\left(C_{[L]}^{n+1}\right)_{+} \backslash S_{[L]}^{n}(0) \\ H(x), & \text { if } x \in\left(\widehat{D_{[L]}^{n+1}}\right)_{-} \backslash S_{[L]}^{n}(0) \\ x_{0}, & \text { if } x \in S_{[L]}^{n}(0)\end{cases}
$$


The mapping $k$ represents the product of $\alpha$ and $\alpha^{-1}$ in the group $\pi_{n+1}^{[L]}\left(Y, y_{0}\right)$ and hence is $[L]$-homotopically trivial.

Let $\mu^{[L]}$ be a strongly $[L]$-universal $\mathrm{AE}([L])$ - -ompactum $^{1}$ of extension dimension $[L]$ provided by Proposition 3.6. According to (iv) of that proposition we may assume that $\mu^{[L]}$ is an $[L]$-polyhedron. Let $i: \widetilde{S_{[L]}^{n+1}} \hookrightarrow \mu^{[L]}$ be an embedding. By Proposition 4.3, there exists an extension $\bar{k}: \mu^{[L]} \rightarrow Y$ of $k$. Applying the RLP of $p$ to the pair $\widetilde{D_{[L]}^{n+1}} \subset \mu^{[L]}$ we obtain a lifting $\bar{K}: Z \rightarrow X$ of $\bar{k}$ which is an extension of $K$. The mapping $F=\left.\bar{K}\right|_{\left(D_{[L]}^{n+1}\right)_{+}}:\left(D_{[L]}^{n+1}\right)_{+} \rightarrow X$ provides a desired extension of $G$. This concludes the proof of the claim.

Let now $S_{[L]}^{n} \subset D_{[L]}^{n+1}$ be a pair of $[L]$-polyhedra $((n+1)$ - $[L]$-disk and corresponding $n$ - $[L]$-sphere) provided by the Claim. Let also $\tau$ denote the correspondent triangulation of $D^{n+1}$ and $f: D_{[L]}^{n+1} \rightarrow D^{n+1}$ be the correspondent approximately $[L]$-soft mapping. Consider $[L]$-dimensional $A E([L])$-space $\mathbb{D}_{[L]}^{n+1}$ which satisfies conditions (i)-(iv) of Proposition 3.6 and the corresponding $[L]$ invertible and approximately $[L]$-soft mapping $f^{[L]}: \mathbb{D}_{[L]}^{n+1} \rightarrow D^{n+1}$. We assume that condition (iv) of Proposition 3.6 is satisfied with respect to the same triangulation $\tau$ of $D^{n+1}$. Put $\mathbb{S}_{[L]}^{n}=\left(f^{[L]}\right)^{-1}\left(S^{n}\right)$. Then $\mathbb{S}_{[L]}^{n} \in A N E([L])$ and the restriction $\left.f^{[L]}\right|_{\mathbb{S}_{[L]}^{n}}$ is also approximately $[L]$-soft.

The further proof consists of two steps. First we apply the RLP of $p$ with respect to the pair $S_{[L]}^{n} \subset D_{[L]}^{n+1}$ to verify that $p$ has RLP with respect to the pair $\left(\mathbb{S}_{[L]}^{n}, \mathbb{D}_{[L]}^{n+1}\right)$. Then we use this fact to show that $p$ has RLP with respect to arbitrary pair of $n$ - $[L]$-sphere and $(n+1)$ - $[L]$-disk.

Since the mapping $f^{[L]}$ is $[L]$-invertible there exists a mapping $\tilde{f}$ such that $f^{[L]} \circ \tilde{f}=f$. Next, Proposition 3.6(iii) provides us with an embedding $g: D_{[L]}^{n+1} \rightarrow$ $\mathbb{D}_{[L]}^{n+1}$ such that

$$
f^{[L]} \circ g=f^{[L]} \circ \tilde{f}=f
$$

It is easy to check that

$$
g\left(S_{[L]}^{n}\right) \subset \mathbb{S}_{[L]}^{n} \text { and } g\left(D_{[L]}^{n+1}\right) \backslash S_{[L]}^{n} \subset \mathbb{D}_{[L]}^{n+1} \backslash \mathbb{S}_{[L]}^{n} .
$$

In what follows we identify compacta $D_{[L]}^{n+1}$ and $S_{[L]}^{n}$ with their images $g\left(D_{[L]}^{n+1}\right)$ and $g\left(S_{[L]}^{n}\right)$. Consider the space $\widetilde{D_{[L]}^{n+1}}=\mathbb{S}_{[L]}^{n} \cup D_{[L]}^{n+1}$. It is not hard to see that $\widetilde{D_{[L]}^{n+1}} \in A E([L])$. Moreover, $\widetilde{D_{[L]}^{n+1}}$ is $(n+1)$ - $[L]$-disk and $[L]$-polyhedron. Let

\footnotetext{
${ }^{1}$ Note that the Hilbert cube $\mathbb{Q}$ is an example of strongly $[\{*\}]$-universal $\mathrm{AE}([\{*\}])$ compactum of extension dimension $[\{*\}]$ and the universal $n$-dimensional Menger compactum $\mu^{n}$ serves as an example of strongly $\left[S^{n}\right]$-universal AE $\left(\left[S^{n}\right]\right)$-compactum of extension dimension $\left[S^{n}\right]$ (see [5, Chapters $\left.2 \& 4\right]$ for a comprehensive discussion of various aspects of strong universality property within the general theory of absolute extensors).
} 
now $F: \mathbb{D}_{[L]}^{n+1} \rightarrow Y$ and $G: \mathbb{S}_{[L]}^{n} \rightarrow X$ be mappings such that $\left.F\right|_{\mathbb{S}_{[L]}^{n}}=p \circ G$. We have to find a lifting $\bar{G}$ of $F$ extending $G$. Since $p$ has RLP with respect to the pair $S_{[L]}^{n} \subset D_{[L]}^{n+1}$ there exists an extension $\widetilde{G}: \widetilde{D_{[L]}^{n+1}} \rightarrow X$ of $G$ such that $F \mid \widetilde{D_{[L]}^{n+1}}=p \circ \widetilde{G}$. Now the right lifting property of $[L]$-fibration applied to the pair $\widetilde{D_{[L]}^{n+1}} \subset \mathbb{D}_{[L]}^{n+1}$ provides us with the desired lifting $\bar{G}$.

Let now $\left(S_{[L]}^{n}, D_{[L]}^{n+1}\right)$ denote an arbitrary pair of $(n+1)$ - $[L]$-disk and $n$ - $[L]-$ sphere. The corresponding approximately $[L]$ soft mapping of $D_{[L]}^{n+1}$ onto $D^{n+1}$ is denoted still by $f$. As above, we can find an embedding $D_{[L]}^{n+1} \hookrightarrow \mathbb{D}_{[L]}^{n+1}$ such that $S_{[L]}^{n} \hookrightarrow \mathbb{S}_{[L]}^{n}$ and $D_{[L]}^{n+1} \backslash S_{[L]}^{n} \hookrightarrow \mathbb{D}_{[L]}^{n+1} \backslash \mathbb{S}_{[L]}^{n}$. Since both mappings $\left.f^{[L]}\right|_{\mathbb{S}_{[L]}^{n}}$ and $\left.f\right|_{S_{[L]}^{n}}$ are approximately $[L]$-soft there exists a retraction $r: \mathbb{S}_{[L]}^{n} \rightarrow S_{[L]}^{n}$ (which is $[L]$-homotopy equivalence). Since $D_{[L]}^{n+1} \in A E([L])$ this retraction can be extended to a retraction $R: \mathbb{D}_{[L]}^{n+1} \rightarrow D_{[L]}^{n+1}$. Consider two mappings $F: D_{[L]}^{n+1} \rightarrow Y$ and $G: S_{[L]}^{n} \rightarrow X$ such that $\left.F\right|_{S_{[L]}^{n}}=p \circ G$. Since, as shown above, the mapping $p$ possesses RLP with respect to the pair $\mathbb{S}_{[L]}^{n} \subset \mathbb{D}_{[L]}^{n+1}$ the mapping $F \circ R: \mathbb{D}_{[L]}^{n+1} \rightarrow Y$ has a lifting $\bar{G}: \mathbb{D}_{[L]}^{n+1} \rightarrow X$ which extends the mapping $G \circ r: \mathbb{S}_{[L]}^{n} \rightarrow X$. Clearly, the restriction $\left.\bar{G}\right|_{D_{[L]}^{n}} \rightarrow X$ is a lifting of $F$ extending $G$, as required. Proof of the necessity is completed.

Now we show that the condition of the Lemma is also sufficient. Let $p: X \rightarrow$ $Y$ be a fibration possessing the RLP with respect to any pair $S_{[L]}^{n} \subset D_{[L]}^{n+1}$ where $D_{[L]}^{n+1}$ is an $(n+1)$ - $[L]$-disk and $S_{[L]}^{n}$ is a corresponding $n$ - $[L]$-sphere, $n=0,1, \ldots$ We need to show that $p$ is acyclic. Fix $n=0,1, \ldots$ and a point $x_{0} \in X$. Let $y_{0}=p\left(x_{0}\right)$. Consider an arbitrary element $\alpha$ of the group $\pi_{n}^{[L]}\left(Y, y_{0}\right)$. Let $\widetilde{S_{[L]}^{n}}=D_{[L]}^{n} / S_{[L]}^{n-1}$ be a quotient space, where $D_{[L]}^{n}$ is an $n$ - $[L]$-disk. Let $\phi$ be the correspondent quotient mapping and denote $s_{[L]}=\phi\left(S_{[L]}^{n-1}\right)$. It is easy to check that compactum $\widetilde{S_{[L]}^{n}}$ admits an approximately $[L]$-soft mapping onto $S^{n}$. Therefore element $\alpha$ can be represented by means of a mapping $\widetilde{f}: \widetilde{S_{[L]}^{n}} \rightarrow Y$ such that $\widetilde{f}\left(s_{[L]}\right)=y_{0}$. The mapping $\widetilde{f}$ allows us to define mapping $f: D_{[L]}^{n+1} \rightarrow Y$ as follows.

$$
f(x)= \begin{cases}\widetilde{f}(x), & \text { if } x \in D_{[L]}^{n} \backslash S_{[L]}^{n-1} \\ x_{0}, & \text { if } x \in S_{[L]}^{n-1}\end{cases}
$$

Let $G: S_{[L]}^{n-1} \rightarrow x_{0}$ be a constant mapping. Applying RLP of $p$ to the pair $S_{[L]}^{n-1} \subset D_{[L]}^{n}$ we can find a lifting $F: D_{[L]}^{n} \rightarrow X$ of $f$ extending $G$. Now we define 
mapping $\widetilde{F}: \widetilde{S_{[L]}^{n}} \rightarrow X$ letting

$$
\widetilde{F}(x)= \begin{cases}F(x), & \text { if } x \in \widetilde{S_{[L]}^{n}} \backslash s_{[L]} \\ x_{0}, & \text { if } x=s_{[L]}\end{cases}
$$

It is easy to see that $\tilde{f}=p \circ \widetilde{F}$. This shows that $p$ induces an epimorphism of $n^{\text {th }}$-[L]-homotopy groups.

Consider now a mapping $F: S_{[L]}^{n} \rightarrow X$ where $S_{[L]}^{n}$ coincides with the subset $S_{[L]}^{n}$ of $(n+1)$ - $[L]$-disk $D_{[L]}^{n+1}$, such that a composition $p \circ F: S_{[L]}^{n} \rightarrow Y$ is $[L]-$ homotopically trivial. Then there exists an extension $g: D_{[L]}^{n+1} \rightarrow Y$ of $p \circ F$. Applying RLP of $p$ to the pair $S_{[L]}^{n} \subset D_{[L]}^{n+1}$ we can find a lifting $G: D_{[L]}^{n+1} \rightarrow X$ of $g$ extending $F$. By Proposition 4.3 the mapping $F$ is also $[L]$-homotopically trivial. This shows that $p$ induces a monomorphism of the $n^{t h}-[L]$-homotopy groups. Therefore the fibration $p$ is acyclic.

The above Lemma allows us to prove the following statement.

Corollary 4.5. Every $[L]$-polyhedron is cofibrant and fibrant object in the category TOP with weak equivalences, fibrations and cofibrations, defined as in Definition 4.2.

Proof. Definition of $[L]$-fibration implies that every topological space is a fibrant object. To prove that every $[L]$-polyhedron $X$ is cofibrant we need to show that for any acyclic fibration $p: A \rightarrow B$ and for any mapping $f: X \rightarrow B$ there exists a lifting $F: X \rightarrow A$. Since any $[L]$-polyhedron can be obtained by attaching $n$ - $[L]$-cells, such a lifting $F$ can be constructed by induction. If $g: X \rightarrow Y$ is an approximately $[L]$-soft mapping corresponding to $X$ (see Definition 3.7 ), then for each $y \in Y^{(0)}$ we have $g^{-1}(y) \in A E([L])$. Therefore we can begin the inductive construction applying RLP of $p$ to the pairs $x \in g^{-1}(y)$ for each $y \in Y^{(0)}$ (the point $x$ is arbitrary). We perform inductive steps applying Lemma 4.4. Finally, the resulting map $F$ is continuous by virtue of the fact that $C$ is closed subspace of $X$ if and only if $C \cap X^{(n)}$ is closed for each $n$ (see remarks following Definition 3.7).

Next we need the following proposition.

Lemma 4.6. Every map $p: X \rightarrow Y$ in TOP can be factored in either of two ways:

(a) $f=p_{\infty} i_{\infty}$, where $i_{\infty}: X \rightarrow X^{\prime}$ is a cofibration and $p_{\infty}: X^{\prime} \rightarrow Y$ is an acyclic $[L]$-fibration.

(b) $f=q_{\infty} j_{\infty}$, where $j_{\infty}: X \rightarrow X^{\prime}$ is a weak $[L]$-homotopy equivalence which has the LLP with respect to $[L]$-fibrations, and $q_{\infty}: X^{\prime} \rightarrow Y$ is a $[L]$ fibration. 
Proof. (a) Let $\mathcal{F}$ be the set of all inclusions $\left\{f_{t}: A_{t} \hookrightarrow B_{t} ; t \in T\right\}$ such that $B_{t}$ is an $n$ - $[L]$ disk and $A_{t}$ is a corresponding $(n-1)$ - $[L]$-sphere, $n=0,1, \ldots$, or $A_{t}$ and $B_{t}$ are finite $[L]$-polyhedra such that both $A_{t}$ and $B_{t}$ are $A E([L])$-spaces. For each $t \in T$ consider the set $S(t)$ which contains all pairs of maps $(g, h)$ such that the following diagram

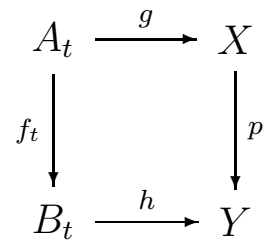

commutes. By gluing a copy of $B_{t}$ to $X$ along $A_{t}$ for every commutative diagram of the above form we obtain the Gluing Construction $G^{1}(\mathcal{F}, p)$ as in the following pushout diagram

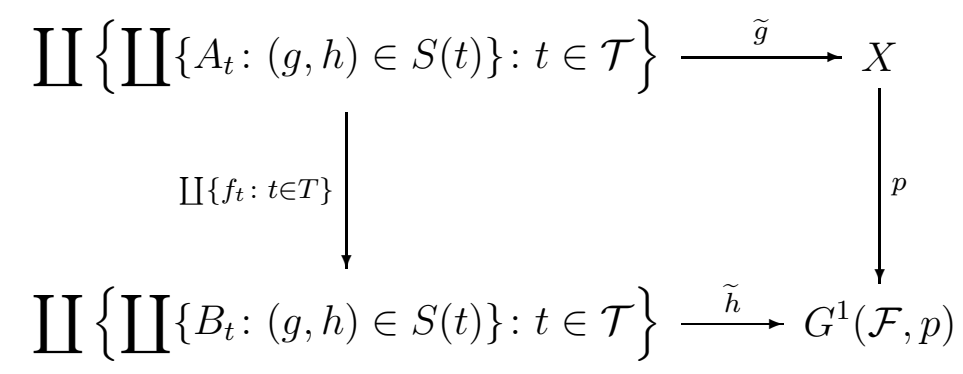

Let $i_{1}: X \rightarrow G^{1}(\mathcal{F}, p)$ denote the natural embedding. By the universal property of colimits, there exists a map $p_{1}: G^{1}(\mathcal{F}, p) \rightarrow Y$ such that $p_{1} i_{1}=p$. By repeating this process we obtain the following infinite commutative diagram

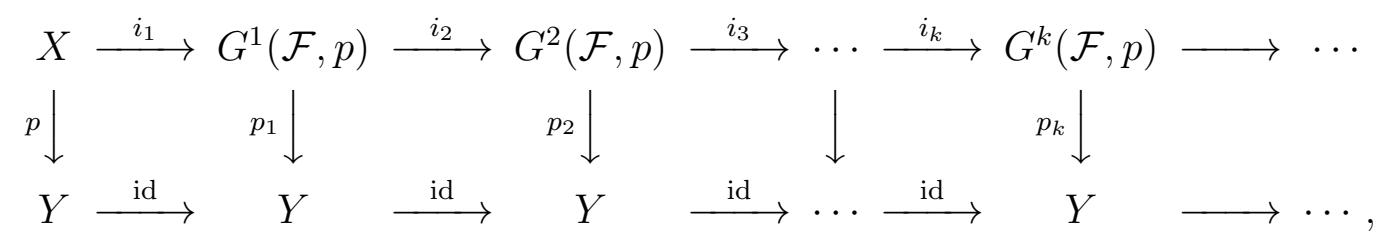

Next consider the colimit $G^{\infty}(\mathcal{F}, p)$ of the upper row (i.e. the Infinite Gluing Construction) in the above diagram. Obviously there are natural maps $i_{\infty}: X \rightarrow G^{\infty}(\mathcal{F}, p)$ and $p_{\infty}: G^{\infty}(\mathcal{F}, p) \rightarrow Y$ such that $p=p_{\infty} i_{\infty}$.

It follows from the small object argument (see [14, Proposition 7.17]) and the proof of [14, Lemma 8.12]) that the map $p_{\infty}: G^{\infty}(\mathcal{F}, p) \rightarrow Y$ has the RLP with 
respect to each of the maps in the family $\mathcal{F}$. This, according to definition and by Lemma 4.4 , means that $p_{\infty}$ is an acyclic $[L]$-fibration.

Since every map $f_{t} \in \mathcal{F}$ is either an inclusion of finite $[L]$-polyhedra, which are $A E([L])$-spaces, or an inclusion of $(n-1)^{t h}$ - $[L]$-sphere into $n^{t h}-[L]$-disk for some $n$, it follows from Definition $4.2(\mathrm{ii})_{L}$ and Lemma 4.4 that $f_{t}$ has the LLP with respect to acyclic $[L]$-fibrations. The colimit universality property of the Infinite Gluing Construction guarantees that the map $i_{\infty}$ also has the LLP with respect to all acyclic $[L]$-fibrations and therefore is a $[L]$-cofibration according to Definition $4.2(\text { iii })_{L}$.

(b) Let $\mathcal{F}$ be the set of all inclusions $\left\{f_{t}: A_{t} \hookrightarrow B_{t} ; t \in T\right\}$ of finite $[L]$ polyhedra such that $A_{t}$ and $B_{t}$ are $A E([L])$-spaces. For each $t \in T$ consider the set $S(t)$ which contains all pairs of maps $(g, h)$ such that the following diagram

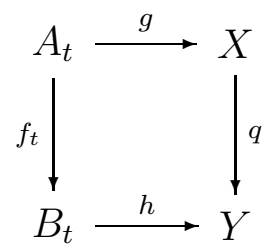

commutes. As in the part (b), we obtain the Infinite Gluing Construction $G^{\infty}(\mathcal{F}, q)$ and natural maps $j_{\infty}: X \rightarrow G^{\infty}(\mathcal{F}, q)$ and $q_{\infty}: G^{\infty}(\mathcal{F}, q) \rightarrow Y$ such that $q=q_{\infty} j_{\infty}$.

It follows from the small object argument (see [14, Proposition 7.17]) and the proof of $\left[14\right.$, Lemma 8.12]) that the map $q_{\infty}: G^{\infty}(\mathcal{F}, q) \rightarrow Y$ has the RLP with respect to each of the maps in the family $\mathcal{F}$. This, according to definition, means that $q_{\infty}$ is a $[L]$-fibration.

Since every map $f_{t} \in \mathcal{F}$ is an inclusion of finite $[L]$-polyhedra which are $A E([L])$-spaces, it follows that $f_{t}$ has the LLP with respect to $[L]$-fibrations (Definition $\left.4.2(\mathrm{ii})_{L}\right)$. It is clear that $j_{\infty}$ is a weak $[L]$-homotopy equivalence. The colimit universality property of the Infinite Gluing Construction implies that the map $j_{\infty}$ also has the LLP with respect to $[L]$-fibrations.

Theorem 4.7. Let $L$ be a finite polyhedron. Then the category TOP with weak equivalences, fibrations and cofibrations, defined as in Definition 4.2, is a model category.

Proof. Axioms (MC1)-(MC2) are trivially satisfied. Next, observe that the notions of $[L]$-fibrations and $[L]$-cofibrations are defined by lifting properties and hence the classes of $[L]$-fibrations and $[L]$-cofibrations are closed by retracts. Further, a retract of an isomorphism is an isomorphism. These facts imply Axiom (MC3). Lemma 4.6 shows that Axiom (MC5) is also satisfied.

In order to verify Axiom (MC4) we need only to check that acyclic $[L]-$ cofibrations have the LLP with respect to all $[L]$-fibrations ([L]-cofibrations 
have the property, required in (MC4), by definition). Consider a commutative diagram

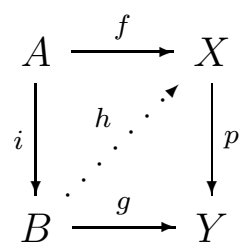

of unbroken arrows, where $i$ is an acyclic $[L]$-cofibration and $p$ is a $[L]$-fibration. We need to show existence of $h$. By Lemma 4.6 we have the following commutative diagram

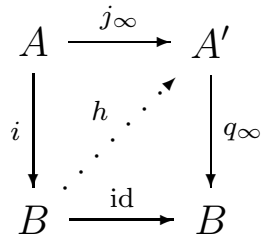

where $j_{\infty}$ is a weak $[L]$-homotopy equivalence having the LLP with respect to all $[L]$-fibrations and $q_{\infty}$ is an $[L]$-fibration.

Since $i$ and $j_{\infty}$ are weak $[L]$-homotopy equivalences it follows from (MC2) that $q_{\infty}$ is a weak $[L]$-homotopy equivalence. Therefore $q_{\infty}$ is an acyclic $[L]-$ fibration. Since $i$ is a $[L]$-cofibration, there exists a lifting $h^{\prime}: B \rightarrow A^{\prime}$ of $q_{\infty}$. Since $j_{\infty}$ has the LLP with respect to $[L]$-fibrations, there exists a lifting $h^{\prime \prime}: A^{\prime} \rightarrow X$ of a composition $g \circ q_{\infty}: A^{\prime} \rightarrow Y$. We let $h=h^{\prime \prime} \circ h^{\prime}$. It is easily seen that $h$ has the required property.

Theorem 4.7 and Corollary 4.5 imply the following important observation.

Corollary 4.8. A map between $[L]$-complexes is an $[L]$-homotopy equivalence if and only if it induces isomorphisms of all $[L]$-homotopy groups.

In conclusion let us show that our notion of $\left[S^{n}\right]$-fibration differs from the usual notion of $n$-fibration. Consequently the model category structure generated by $L=S^{n}$ in theorem 4.7 differs from the one described in Theorem C (note, however, that these two structures have identical weak equivalences). It is unclear if the model category structure generated on TOP by $L=\{$ point coincides with the one described in Theorem A (although classes of weak equivalences are identical and the notion of $[L]$-homotopy coincides with the notion of usual homotopy in this case). 
To this end let $n \geq 0$ and let $X$ and $Y$ be copies of the $n$-dimensional universal Menger compactum $\mu^{n}$. Consider the Dranishnikov's resolution $p: X \rightarrow Y$ constructed in [13] (see also [5, §4.2]). Observe that the map $p$, being polyhedrally $n$-soft, is an acyclic $n$-fibration. Note that $p$ is $(n-1)$-soft, but not $n$-soft. The latter means that there exists an at most $n$-dimensional compactum $B_{0}$, a closed embedding $i_{0}: A_{0} \hookrightarrow B_{0}$ and two maps $\alpha: A_{0} \rightarrow X$ and $\beta: B_{0} \rightarrow Y$ such that there is no lifting of $\beta$, extending $\alpha$. In other words, the following commutative diagram of unbroken arrows

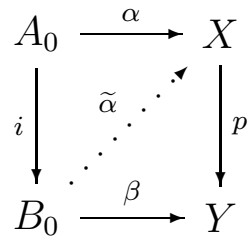

cannot be completed by the broken arrow.

Let $A$ and $B$ denote two additional copies of the Menger compactum $\mu^{n}$ and let $i: A \rightarrow B$ be a $Z$-embedding. Consider embeddings $j_{0}: A_{0} \rightarrow A$ and $j: B_{0} \rightarrow B$ such that $j \circ i_{0}=i \circ j_{0}$ and $j\left(B_{0} \backslash i_{0}\left(A_{0}\right)\right) \subseteq B \backslash i(A)$ (embeddings with the indicated properties exist because $i$ is a $Z$-embedding). Since $X$ is an $\mathrm{AE}(n)$-compactum and $\operatorname{dim} A=n$, it follows that there exists a map $g: A \rightarrow X$ such that $\alpha=g \circ i_{0}$. Define the map $h: i(A) \cup j\left(B_{0}\right) \rightarrow Y$ by letting

$$
h(b)= \begin{cases}p\left(g\left(i^{-1}(b)\right)\right), & \text { if } b \in i(A), \\ \beta\left(j^{-1}(b)\right), & \text { if } b \in j\left(B_{0}\right) .\end{cases}
$$

Since $Y \in \operatorname{AE}(n)$ and $\operatorname{dim} B=n$, there exists a map $f: B \rightarrow Y$ such that $f \mid\left(i(A) \cup j\left(B_{0}\right)\right)=h$. Note that $p \circ g=f \circ i$. 


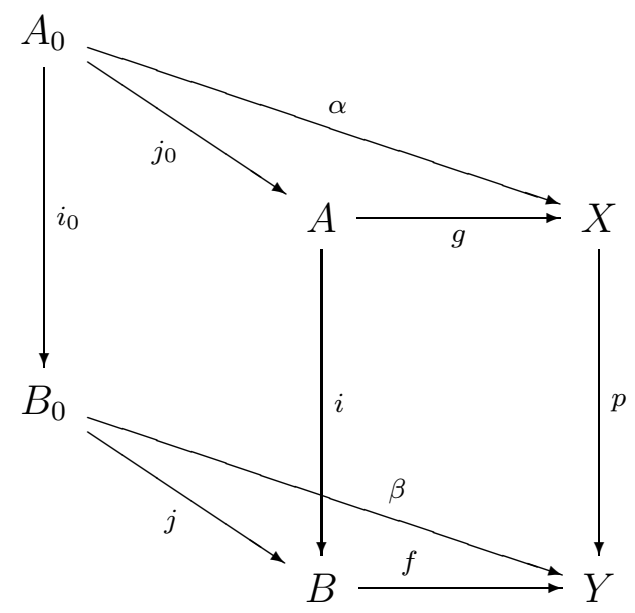

Finally observe that the map $f$ does not have a lifting extending $g$, since this would imply existence of lifting of $\beta$ extending $\alpha$. Hence $p$ is not an $\left[S^{n}\right]-$ fibration.

\section{REFERENCES}

[1] H. J. Bauess, Algebraic Homotopy, Cambridge Univ. Press, Cambridge, 1989.

[2] M. Bestvina, Characterizing k-dimensional universal Menger compacta, Memoirs Amer. Math. Soc. No. 380, 71 (1988).

[3] K. S. Brown, Abstract homotopy theory and generalized sheaf cohomology, Trans. Amer. Math. Soc. 186 (1973), 419-458.

[4] A. Chigogidze, Infinite Dimensional Topology and Shape Theory, in: Handbook of Geometric Topology (eds.: R. Daverman, R. B. Sher), North-Holland, Amsterdam, 2001, 307-371.

[5] A. Chigogidze, Inverse Spectra, North Holland, Amsterdam, 1996.

[6] A. Chigogidze, The theory of n-shapes, Russian Math. Surveys 44 (1989), 145-174.

[7] A. Chigogidze, Compacta lying in the $n$-dimensional universal Menger compactum and having homeomorphic complements in it, Math. USSR Sb. 61 (1988), 471-484.

[8] A. Chigogidze, V. V. Fedorchuk, Menger(Nöbeling) manifolds versus Hilbert cube (space) manifolds - a categorical comparison, Monatsh. Math. 130, No.2, (2000), 89-97.

[9] A. N. Dranishnikov, The Eilenberg-Borsuk theorem for mappings in an arbitrary complex, Matem. Sb. 185 (1994), 81-90.

[10] A. N. Dranishnikov, On a problem of P. S. Alexandrov, Matem. Sb. 135 (1988), 551-557.

[11] A. Dranishnikov, J. Dydak, Extension dimension and extension types, Proc. Steklov Inst. Math. 212, no.1 (1996), 55-88

[12] A. N. Dranishnikov and D. Repovš, Cohomological dimension with respect to perfect groups, Topology Appl. 74(1996), 123-140

[13] A. N. Dranishnikov, Universal Menger compacta and universal mappings, Matem. Sb. 129 (1986), 121-139.

[14] W. G. Dwyer, J. Spalinski, Homotopy Theories and Model Categories, in: Handbook of Algebraic Topology (ed. I. M. James), North Holland, Amsterdam, 1995, 73-126. 
[15] J. Dydak and J. Walsh, Infinite-dimensional compacta having cohomological dimension two: an application of the Sullivan conjecture, Topology 32(1993), 93-104.

[16] C. Elvira-Donazar, L. J. Hernandez-Paricio, Closed model categories for the n-types of spaces and simplicial sets, Math. Proc. Cambridge Philos. Soc. 118 (1995), no. 1, 93-103.

[17] R. H. Fox, On the Lusternik-Schnirelman category, Ann. of Math. 42 (1941), 333-370.

[18] R. Fritsch, R. A. Piccinini, Cellular Structures in Topology, Cambridge Univ. Press, Cambridge, 1990.

[19] A. Heller, Stable homotopy categories, Bull. Amer. Math. Soc. 74 (1968), 28-63.

[20] M. Hovey, Model Categories, Amer. Math. Soc. Mathematical Surveys and Monographs, v. $63,1998$.

[21] D. M. Kan, Abstract homotopy theory I, Proc. Nat. Acad. Sci. USA 41 (1955), 1092-1096.

[22] D. M. Kan, Abstract homotopy theory II, Proc. Nat. Acad. Sci. USA 42 (1956), 255-258.

[23] A. Karasev, On $[L]$-homotopy groups, JP Journal of Geometry and Topology, 1, No. 3 (2001), 301-310.

[24] J. L. Loday, Spaces with finitely many homotopy groups, J. Pure Appl. Algebra 34 (1984), $155-178$.

[25] D. Quillen, Homotopical Algebra, Lect. Notes in Math. 43, 1967.

[26] A. Strom, The homotopy category is a homotopy category, Arch. Math. 23 (1972), 435441.

[27] R. M. Switzer, Algebraic Topology - Homotopy and Homology, Springer Verlag, Berlin, 1975.

[28] J. H. C. Whitehead, On the homotopy type of ANR's, Bull. Amer. Math. Soc. 54 (1948), $1133-1145$.

[29] J. H. C. Whitehead, Combinatorial homotopy I, Bull. Amer. Math. Soc. 55 (1949), 213245.

[30] J. H. C. Whitehead, Combinatorial homotopy II, Bull. Amer. Math. Soc. 55 (1949), 453-496.

Department of Mathematics and Statistics, University of Saskatchewan, Mclean Hall, 106 Wiggins Road, Saskatoon, SK, S7N 5E6, Canada

E-mail address: chigogid@math.usask.ca

Department of Mathematics and Statistics, University of Saskatchewan, McLean Hall, 106 Wiggins Road, Saskatoon, SK, S7N 5E6, Canada

E-mail address: karasev@math.usask.ca 